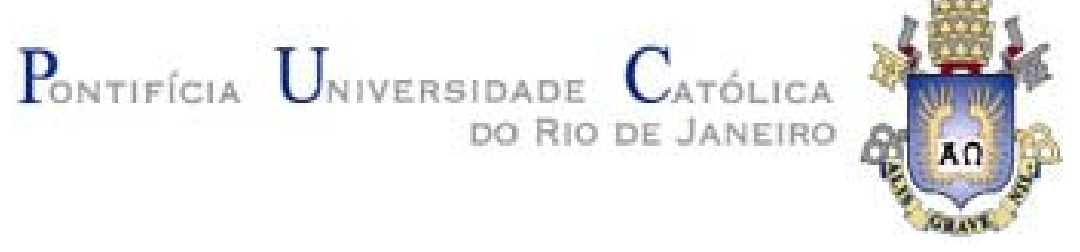

Walter Eduardo Lazo Málaga

Obtenção do Óxido de Cromo a Partir de
Finos de uma Liga FeCr-alto carbono

Dissertação de Mestrado

Dissertação apresentada como requisito parcial para obtenção do Título de Mestre em Engenharia Metalúrgica e de Materiais do Programa de Pósgraduação em Engenharia de Materiais e de Processos Químicos e Metalúrgicos da PUC-Rio.

Orientador: Prof. Eduardo de Albuquerque Brocchi

Rio de Janeiro Setembro de 2009 
Walter Eduardo Lazo Málaga

\section{Obtenção do Óxido de Cromo a Partir de Finos de uma Liga FeCr-alto Carbono}

Dissertação apresentada como requisito parcial para obtenção do Título de Mestre pelo Programa de PósGraduação de Engenharia de Materiais e de Processos Químicos e Metalúrgicos da PUC-Rio. Aprovada pela Comissão Examinadora abaixo assinada.

Dr. Eduardo de Albuquerque Brocchi Orientador e Presidente Pontifícia Universidade Católica do Rio de Janeiro - PUC-Rio

Dr. Francisco José Moura

Pontifícia Universidade Católica do Rio de Janeiro - PUC-Rio

Dr. Olavo Barbosa Filho

Universidade do Estado do Rio de Janeiro - UERJ

Prof. José Eugênio Leal

Coordenador Setorial de Pós-Graduação do Centro Técnico Científico da PUC - Rio

Rio de Janeiro, 11 de Setembro de 2009 
Todos os direitos reservados. É proibida a reprodução total ou parcial do trabalho sem autorização da universidade, do autor e do orientador.

\section{Walter Eduardo Lazo Málaga}

Graduou-se em Engenharia Metalúrgica pela UNSA (Universidad Nacional de San Agustin), Arequipa-Perú (2002) e é Técnico em Mecânica de Produção pelo ISPPD (Instituto Superior Pedro P. Diaz), Arequipa-Perú (1995).

Ficha Catalográfica

Lazo Málaga, Walter Eduardo

Obtenção do óxido de cromo a partir de finos de uma liga FeCr-alto carbono / Walter Eduardo Lazo Málaga ; orientador: Eduardo de Albuquerque Brocchi. - 2009.

73 f. : il.(color.) ; $30 \mathrm{~cm}$

Dissertação (Mestrado em Engenharia de Materiais)-Pontifícia Universidade Católica do Rio de Janeiro, Rio de Janeiro, 2009.

Inclui bibliografia

1. Engenharia de materiais - Teses. 2. Ustulação alcalina. 3. Óxido de cromo. I. Brocchi, Eduardo de Albuquerque. II. Pontifícia Universidade Católica do Rio de Janeiro. Departamento de Engenharia de Materiais. III. Título. 
A meus pais pelo apoio incondicional 


\section{Agradecimentos}

A meus pais e irmãos pelo apoio.

Ao professor Eduardo Brocchi pela orientação, parceria, apoio e paciência para a realização deste trabalho.

A aluna Amanda Brandão por toda a imprescindível colaboração e solidariedade.

Aos meus amigos da PUC-Rio.

Ao CNPq pelo apoio financeiro para este trabalho. 


\section{Resumo}

Málaga, Walter Eduardo Lazo; Brocchi, Eduardo de Albuquerque. Obtenção do Óxido de Cromo a Partir de finos de uma Liga FeCr-alto Carbono. Rio de Janeiro, 2009. 73p. Dissertação de Mestrado Departamento de Ciência dos Materiais e Metalurgia, Pontifícia Universidade Católica do Rio de Janeiro.

O trabalho tem como motivação principal conciliar a crescente demanda de cromo em aplicações específicas com o aproveitamento de um resíduo industrial que está na forma de finos de baixo valor agregado. Desta forma, se estuda a possibilidade de recuperar o cromo, na forma de óxido, que está contido em finos da liga Fe-Cr Alto Carbono. Inicialmente, foi estudado o efeito das variáveis relevantes (temperatura, proporção dos reagentes) sobre a ustulação da liga com os hidróxidos de $\mathrm{Na}$ e $\mathrm{Mg}$. Os resultados foram baseados na solubilização do produto ustulado e a subseqüente obtenção de uma solução rica em cromo separada de um composto rico em ferro. A etapa final envolve a precipitação do óxido de cromo. Os materiais envolvidos foram caracterizados por análise química, difração de raio-X e microscopia eletrônica de varredura. Os resultados qualitativos indicam a viabilidade do processo, mas, condições otimizadas devem ser identificadas visando uma maior eficiência do mesmo.

\section{Palavras-chave}

Ustulação alcalina; óxido de cromo. 


\section{Abstract}

Málaga, Walter Eduardo Lazo; Brocchi, Eduardo de Albuquerque. (Advisor). Obtaining Chromium Oxide from fine $\mathrm{FeCr}$ high-carbon alloy. Rio de Janeiro, 2009. 73p. MSc. Dissertation - Departamento de Ciência dos Materiais e Metalurgia, Pontifícia Universidade Católica do Rio de Janeiro.

The investigation main motivation is to combine the growing chromium demand for specific applications with the reuse of a low value $\mathrm{Cr}-\mathrm{Fe}$-carbon alloy. Thus, it has been studied the possibility of recovering chromium, in the oxide form, from fines generated in the production of $\mathrm{Fe}-\mathrm{Cr}$ High Carbon. Initially, the effect of relevant variables (temperature, ratio of reactants) on the alloy roasting with $\mathrm{Na}$ and $\mathrm{Mg}$ hydroxides, were studied. Then, the chromate solubilization followed by the chromium oxide precipitation have been carried out. The materials involved in the work have characterized by chemical analysis, DRX and MEV/EDS. The qualitative results indicated that the method can be applied but further experiments has to be carried out to optimize the process.

\section{Keywords}

Alkaline roasting; chromium oxide. 


\section{Sumário}

1 INTRODUÇÃO

3 REVISÃO BIBLIOGRÁFICA E FUNDAMENTAÇÃO TEÓRICA

3.1. Processos de obtenção do óxido de cromo $\left(\mathrm{Cr}_{2} \mathrm{O}_{3}\right)$ a partir da cromita $\left(\mathrm{FeO} . \mathrm{Cr}_{2} \mathrm{O}_{3}\right)$ :

3.1.1. Processo tradicional (Ustulação - solubilização seletiva precipitação do hidróxido/ calcinação)

3.1.2. Processo tradicional (Ustulação - solubilização seletiva precipitação do óxido)

3.1.3. Processo baseado nos princípios de produção limpa e indústria ecológica (Ustulação - solubilização seletiva - reação aquecida com carbono)

3.2. Processos de obtenção do óxido de cromo $\left(\mathrm{Cr}_{2} \mathrm{O}_{3}\right)$ a partir dos finos da liga de FeCrAC:

3.2.1. Diagrama $\Delta \mathrm{G}^{\circ} \times \mathrm{T}$

3.2.2. Diagrama da Composição de Equilíbrio

3.2.3. Variação de massa durante a Ustulação

3.2.4. Solubilização

3.2.5. Precipitação Seletiva

4.1. Metodologia Experimental 34

4.2. Ustulação $\quad 35$

4.2.1. Parâmetros Operacionais $\quad 37$

4.3. Solubilização 38

4.4. Filtragem 38

4.5. Precipitação do hidróxido de cromo (III) 41

4.6. Métodos Analíticos, análise e caracterização da amostra 45

4.6.1. Analise Granulométrica $\quad 45$

4.6.2. Difratometria de Raios X (DR-X) 45 
5 RESULTADOS E DISCUSSÕES $\quad 47$

5.1. Caracterização da liga de ferro e cromo com alto teor de carbono $\quad 47$

5.1.1. Análise Granulométrica $\quad 47$

5.1.2. Difração de Raios-X da liga 48

5.1.3. Microscopia Eletrônica de Varredura (MEV/EDS) da liga 49

5.2. Processamento químico do resíduo 52

5.2.1. Ustulacão / Solubilização 52

5.2.2. Precipitação seletiva $\quad 63$

$\begin{array}{ll}6 \text { CONCLUSÕES } & 70\end{array}$

7 REFERÊNCIAS BIBLIOGRÁFICAS 


\section{Lista de tabelas}

Tabela 1 - $\Delta \mathrm{G}^{\circ}$ para cada reação, na faixa de temperatura entre $0^{\circ} \mathrm{C} \mathrm{e}$ $1200^{\circ} \mathrm{C}$, com o reagente $\mathrm{NaOH}$.

Tabela 2 - $\Delta \mathrm{G}^{\circ}$ para cada reação, na faixa de temperatura entre $0^{\circ} \mathrm{C} \mathrm{e}$ $1200^{\circ} \mathrm{C}$, com o reagente $\mathrm{Mg}(\mathrm{OH})_{2} \quad 26$

Tabela 3 - Variação de massa da amostra utilizando $\mathrm{NaOH}$. 30

Tabela 4 - Variação de massa da amostra utilizando $\mathrm{Mg}(\mathrm{OH})_{2}$. 31

Tabela 5 - Distribuição granulométrica do resíduo FeCrAC 47

Tabela 6 - Avaliação quantitativa referente ao Espectro da Figura 27

Tabela 7 - Tabela de reprodutibilidade a $500^{\circ} \mathrm{C}$ e $700^{\circ} \mathrm{C}$

Tabela 8 - Porcentagem de perda de peso usando os reagente com uma relação de $47,5 \%$ FeCr para $52,5 \% \mathrm{NaOH}$ a 500, 600, 700 e $800^{\circ} \mathrm{C} \quad 54$

Tabela 9 - Solubilização com $\mathrm{NaOH}$ com uma relação de

$47,5 \mathrm{FeCrAC} / 2,5 \mathrm{NaOH}$ a $500^{\circ} \mathrm{C}, 600^{\circ} \mathrm{C}, 700^{\circ} \mathrm{C}$ e $800^{\circ} \mathrm{C}$

Tabela 10 - Dados referentes à solubilização com $\mathrm{NaOH}$ e à massa

restante após a filtração da solução.

Tabela 11 - Porcentagem de perda de peso com diferentes

relações de reagentes a $500^{\circ} \mathrm{C}, 600^{\circ} \mathrm{C}$ e $700^{\circ} \mathrm{C} \quad 58$

Tabela 12 - Solubilização com $\mathrm{Mg}(\mathrm{OH})$ com uma relação de $65 \%$

$\mathrm{FeCrAC} / 35 \% \mathrm{Mg}(\mathrm{OH})_{2}$ a $500,600,700^{\circ} \mathrm{C}$

Tabela 13 - Solubilização com $\mathrm{Mg}(\mathrm{OH})_{2}$ com uma relação de $71 \%$

FeCrAC / $29 \% \mathrm{Mg}(\mathrm{OH})_{2}$ a $500,600,700$ e $800^{\circ} \mathrm{C} \quad 60$

Tabela 14 - Perda de peso da amostra, a $500^{\circ} \mathrm{C}$, no decorrer do tempo 61

Tabela 15 - Perda de peso da amostra, a $600^{\circ} \mathrm{C}$, no decorrer do tempo 62

Tabela 16 - Perda de peso da amostra, a $700^{\circ} \mathrm{C}$, no decorrer do tempo 62

Tabela 17 - Perda de peso da amostra, a $800^{\circ} \mathrm{C}$, no decorrer do tempo 62

Tabela 18 - Dados referentes à solubilização com $\mathrm{NaOH}$ e ao processo de precipitação do óxido de cromo $\quad 64$

Tabela 19 - Avaliação quantitativa referente ao espectro da figura $36 \quad 66$

Tabela 20 - Avaliação quantitativa referente ao espectro da figura $39 \quad 68$ 


\section{Lista de Figuras}

Figura 1 - Diagrama de Ellingham para o reagente $\mathrm{NaOH}$

Figura 2 - Diagrama de Ellingham para o reagente $\mathrm{Mg}(\mathrm{OH})_{2}$. 27

Figura 3 - Diagrama de Ellingham para as duas reações de ustulação 27

Figura 4 - Diagrama de distribuição das espécies com $\mathrm{NaOH}$. 29

Figura 5 - Diagrama de distribuição das espécies com $\mathrm{Mg}(\mathrm{OH})_{2}$. 29

Figura 6 - Diagrama Eh X Ph para o Sistema $\mathrm{Cr}-\mathrm{H}_{2} \mathrm{O}$

Figura 7 - Fluxograma geral do processo experimental com o uso de $\mathrm{NaOH}$ na ustulação 34

Figura 8 - Representação esquemática da aparelhagem experimental 36

Figura 9 - Disposição do equipamento na linha de ustulação 37

Figura 10 - disposição da amostra dentro do forno 37

Figura 11 - (a) Solubilização com Agitador magnético usando $\mathrm{NaOH}$; (b)

Solubilização com Agitador magnético usando $\mathrm{Mg}(\mathrm{OH})_{2}$

Figura 12 - Disposição do equipamento na linha de filtragem 39

Figura 13 - Disposição da amostra na estufa 39

Figura 14 - $\left(a_{1}\right)$ Resíduo de Hematita depois da filtragem com $\mathrm{NaOH} ;\left(a_{2}\right)$

resíduo de hematita calcinado; $\left(b_{1}\right)$ Resíduo de Hematita depois da filtragem com $\mathrm{Mg}(\mathrm{OH})_{2} ;\left(\mathrm{b}_{2}\right)$ resíduo de hematita calcinado 40

Figura 15 - a) Solução de cromato de sódio depois da filtragem; b) Solução de cromato de magnésio depois da filtragem 40

Figura 16 - Solução com cromato dissolvido, depois da adição de $\mathrm{H}_{2} \mathrm{SO}_{4}$, com pH igual a 0,97 41

Figura 17 - Adição de peróxido de hidrogênio $\left(\mathrm{H}_{2} \mathrm{O}_{2}\right)$ à solução 42

Figura 18 - Medição do $\mathrm{H}_{2} \mathrm{O}_{2}$ residual 42

Figura 19 - Solução com pH igual a 9,05 depois da adição de hidróxido de sódio $(\mathrm{NaOH}) \quad 43$

Figura 20 - Precipitação do hidróxido de cromo (III) [Cr(OH) $\left.)_{3}\right] 43$

Figura 21 - Solução sendo filtrada 44

Figura 22 - $\mathrm{Cr}(\mathrm{OH})_{3}$ em barquetes, no forno, para ser calcinado 44

Figura 23 - Óxido de cromo $\left(\mathrm{Cr}_{2} \mathrm{O}_{3}\right) \quad 44$

Figura 24 - Liga de FeCr-alto C em pó 45

Figura 25 - Difração de Raios-X (DRX) FeCrAC 48

Figura 26 - Micrografia da liga FeCrAC por MEV 49 
Figura 27 - Análise por EDS da liga FeCrAC

Figura 28 - Resultados quantitativos do EDS

Figura 29 - Diagrama de reprodutibilidade a $500^{\circ} \mathrm{C}$ e $700^{\circ} \mathrm{C}$

Figura 30 - Porcentagem de perda de peso $\mathrm{NaOH}$ com uma relação de $47,5 \% \mathrm{FeCr} / 52,5 \% \mathrm{NaOH}$ a $500^{\circ} \mathrm{C}, 600^{\circ} \mathrm{C}, 700^{\circ} \mathrm{C}$ e $800^{\circ} \mathrm{C}$

Figura 31 - Gráfico comparativo entre a massa da amostra antes e depois do teste de solubilização para as temperaturas de ustulação de 500,600 , 700 e $800^{\circ} \mathrm{C}$ com $\mathrm{NaOH}$

Figura 32 - Diagrama de perda de peso com diferentes relações de reagentes de $\mathrm{FeCrAC} / \mathrm{Mg}(\mathrm{OH})_{2}$ a $500^{\circ} \mathrm{C}, 600^{\circ} \mathrm{C}, 700^{\circ} \mathrm{C}$ e $800^{\circ} \mathrm{C}$

Figura 33 - Gráfico comparativo entre a massa da amostra antes e depois do teste de solubilização para as temperaturas de ustulação de 500, 600, 700 e $800^{\circ} \mathrm{C}$ com $\mathrm{Mg}(\mathrm{OH})_{2}$

Figura 34 - Diagrama da Curva Cinética com 0,5 FeCrAC/ 0,5 $\mathrm{Mg}(\mathrm{OH})_{2}$ a $500,600,700 \mathrm{C}$ e $800^{\circ} \mathrm{C}$

Figura 35 - Micrografia (MEV) do resíduo não solubilizado 65

Figura 36 - Gráfico de EDS referente ao resíduo não solubilizado 65

Figura 37 - Difratograma do resíduo não solubilizado. 66

Figura 38 - Micrografia (MEV) do produto gerado na calcinação 67

Figura 39 - Gráfico de EDS referente ao produto gerado na calcinação 68

Figura 40 - Difratograma do produto da calcinação 